\title{
Decisional Conflict During Major Medical Treatment Decision-making: a Survey Study
}

\author{
Kristen E. Pecanac, PhD, RND, Roger L. Brown, PhD, and Hanna B. Kremsreiter
}

BACKGROUND: Both patients and surrogate decisionmakers experience decisional conflict when making a major medical treatment decision with life or death implications. The relationship between health literacy and decisional conflict while making a major medical treatment decision is not understood.

OBJECTIVE: To identify the prevalence of individuals making major medical treatment decisions for themselves or someone else and to explore the relationships between decisional conflict and circumstances of the decision as well as the decision-maker.

DESIGN: Two-phase survey study: in phase 1, we screened for who made a major treatment decision; in phase 2, we asked eligible respondents about their experience making the decision.

PARTICIPANTS: Address-based random sample of 4000 Wisconsin residents; 1072 completed phase 1 and 464 completed phase 2 .

MAIN MEASURES: We asked respondents about types of decisions made, the most difficult decision made, and characteristics of the decision-maker and the decision. We included the Decisional Conflict Scale and four domains of the Health Literacy Questionnaire. Openended questions also allowed respondents to describe their experiences.

KEY RESULTS: About 43\% of respondents reported making a major medical treatment decision. Decisions about major surgery and life support were regarded as the most difficult decisions. Respondents who made the decision for a spouse/partner $(\beta=6.65, p=0.012)$, parent $(\beta=9.27$, $p<0.001)$, or someone else $(\beta=10.7, p<0.001)$ had higher decisional conflict. Respondents who reported higher ability to actively engage with healthcare providers $(\beta=-$ 5.24, $p=0.002$ ) and to understand health information well enough to know what to do $(\beta=-6.12, p=0.001)$ had lower decisional conflict.

CONCLUSIONS: The need to make major treatment decisions is likely to increase and making decisions on someone else's behalf appeared to be especially difficult. Improving communication to encourage patient and family engagement in the decision-making conversation, particularly for individuals with limited health literacy, may be helpful.

KEY WORDS: decisional conflict; decision-making; health literacy; survey

Electronic supplementary material The online version of this article (https://doi.org/10.1007/s11606-020-06125-1) contains supplementary material, which is available to authorized users.

Received April 15, 2020

Accepted August 10, 2020

Published online August 17, 2020
J Gen Intern Med 36(1):55-61

DOI: $10.1007 / \mathrm{s} 11606-020-06125-1$

(C) Society of General Internal Medicine 2020

$\mathrm{T}$ he AMA Code of Medical Ethics asserts that patients or patient surrogates should be empowered to make decisions about medical treatment even when those decisions are expected to lead to death. ${ }^{1}$ However, making life-or-death decisions about medical treatment is frequently associated with decisional conflict: ${ }^{2,3}$ of feeling uncertain, unclear of values, unsupported, uninformed, and unsatisfied with the decision. ${ }^{4}$ In addition to being burdensome, these major medical treatment decisions may also be prevalent. A study of older adults found about $40 \%$ required a treatment decision before death, of whom $70 \%$ required a surrogate to make that decision. ${ }^{5}$ The need to make major medical treatment decisions is likely greater, however, when considering all individuals, not just older adult decedents. Given the known difficulty in making medical treatment decisions, it is important to determine the extent of treatment decision-making and identify the factors that are best suited for interventions designed to reduce decisional conflict and improve decision-makers' experiences.

Previous studies of treatment decision-making have found certain factors to be associated with lower decisional conflict, such as previous advance care planning ${ }^{6}$ and involvement in decision-making. ${ }^{7,8}$ However, it is not clear how contextual or personal characteristics affect these factors. For example, it is unclear whether the decision-makers' health literacy-a construct that includes ability to appraise information and engage with healthcare providers ${ }^{9}$ - influences decisional conflict. As a result of this overall lack of knowledge and data, a comprehensive view of major medical treatment decisions and the decision-makers using both quantitative and qualitative methods is warranted. The objective of this study is twofold: (1) identify the prevalence of individuals making major medical treatment decisions for themselves or someone else and (2) examine the nature of major medical treatment decision-making, including the predictors of decisional conflict. 


\section{METHODS}

We developed a mail survey in collaboration with the University of Wisconsin Survey Center, which provided question design expertise, obtained the address-based sample of Wisconsin residents, and coordinated data collection. We recruited in two phases: in phase 1, we screened for who made a major treatment decision; in phase 2 , we asked respondents who made decisions about their experiences.

\section{Phase 1}

Phase 1 was a 2-page questionnaire mailed to a random sample of 4000 Wisconsin addresses in January 2019. We asked three questions about whether the respondent had been involved in decision-making about: (1) the use of a specific treatment (such as a feeding tube, a breathing machine, or dialysis), (2) whether or not to receive care (such as hospitalization or resusCitation), or (3) whether or not to engage in hospice care or comfort care. Response options included "yes," "no," or "unsure." Respondents who indicated "yes" or "unsure" to making any of the decisions for themselves or someone else and were at least 18 years old were eligible for phase 2. We also asked for respondents' age, gender, race/ethnicity, highest level of education, as well as for their name and address to ensure that, if eligible, the person who completed phase 1 would be the same person at that address to complete phase 2 .

\section{Phase 2}

Phase 2 was a 10-page questionnaire, including 18 items related to experience making a treatment decision. We asked about making decisions for themselves or for someone else regarding the use of dialysis, feeding tubes/artificial nutrition or hydration, ventilators, resuscitation, hospitalization, palliative care, hospice care, major surgery, or organ transplant. We chose these options because they reflected major treatment decisions with potential life or death implications expected for patients experiencing a chronic illness or an acute but critical insult. Each respondent was able to select multiple decisions. Respondents could also share a major treatment decision that was not included in the options.

Of all the decisions that respondents had made for themselves or someone else, they were directed to indicate the most difficult decision, what the choices were, and which choice they made. We had respondents write-in this response to ensure accurate capture of the type of decision and allow for description of the choices, which involved high-stakes outcomes, such as survival. We grouped responses into seven categories: life support, institutionalization, life-prolonging cancer treatment, hospice, surgery, code status, and other. Respondents answered questions about the difficult decision: how long ago the decision was made, who they made the decision for (including themselves, if indicated), and how old was the person for whom they made the decision. To further understand the experience of treatment decision-making, we included two open-ended questions: "Please describe what you remember most about making this decision" and "Is there anything else you want to share, or that you think we should know, about making this decision?"

To assess for decisional conflict, we used the Decisional Conflict Scale. The Decisional Conflict Scale is sixteen items over five domains: feeling informed, feeling clear about their values, feeling supported, feeling certain, and feeling they were making an effective decision. ${ }^{4}$ The Decisional Conflict Scale is valid and reliable for individuals making treatment decisions, including end-of-life decisions for themselves ${ }^{10}$ or for someone else. ${ }^{11}$

To assess for health literacy, we used four of the nine domains of the Health Literacy Questionnaire-social support for health, appraisal of health information, ability to actively engage with healthcare providers, and ability to understand health information well enough to know what to do. Due to concerns for the questionnaire being too long and diminishing participation in the study, we decided to include only the subscales that seemed to be most relevant to making major medical treatment decisions. The four subscales are valid and reliable, and higher scores indicate higher health literacy for that domain (there is no composite score). ${ }^{9}$

To encourage enrollment, we included a $\$ 1$ pre-incentive in the first mailing of phase 1 and $\$ 5$ in the first mailing of phase 2. For both phases, we sent reminder postcards and a second mailing of the questionnaires to the addresses that did not respond to the first mailing. We closed enrollment for phase 1 at the end of April 2019 and for phase 2 at the beginning of August 2019 due to declining return of surveys.

This study was reviewed and approved by the Health Sciences Institutional Review Board. Respondents completing and returning the surveys implied consent.

We encountered missing data. Per guidance on the use of the Health Literacy Questionnaire, we only scored 5-question subscales that had 3 or more questions answered. There were 23 surveys that had less than 3 questions answered in a subscale and consequently these subscales were not scored. For the Decisional Conflict Scale, we only scored the scale if at least 10 of the 16 questions were answered. There were 54 surveys that had less than 10 questions answered, and therefore the scale was not scored. While there are different subscales of the Decisional Conflict Scale, we only calculated the composite score as our outcome variable.

\section{Quantitative Data Analysis}

We used descriptive statistics to provide an overview of the respondents and treatment decisions made. We used regression to examine predictors for decisional conflict in making the most difficult decision. Due to the number of potential predictors (race/ethnicity, age, level of education, gender, and four aspects of health literacy of the decision-maker, age of the person for whom the decision was made, relationship to the 
decision-maker [including oneself], time since the decision was made, and the type of decision), we used lasso regression to select variables for best fit. The lasso shrinks some coefficients and sets others to zero, making the model more easily interpretable with more stable prediction accuracy. ${ }^{12}$ Crossvalidation was used in lasso regression for variable selection and compared with alternate methods (e.g., lowest BIC and multiple lasso adaptive) to indicate the better approach. After determining which variables to retain in the model, we ran a linear regression model of their effect on decisional conflict. We used Stata 16 for all analyses.

\section{Qualitative Data Analysis}

We used inductive content analysis ${ }^{13}$ to analyze the openended questions. Two authors (KP, HK) read the responses to the open-ended questions and coded the data inductively-without a framework guiding the coding - developing the codebook and adding codes as necessary. We analyzed all surveys together, coming to consensus for each code, to reduce individual bias. We then put codes that appeared to "belong" to the same group into categories and abstracted for higher-level analysis. ${ }^{13} \mathrm{We}$ analyzed responses in all surveys, instead of relying on determination of thematic saturation to determine sample size. While we continued to uncover new experiences in the responses, those experiences fit with our codes and we came to a point in the coding process that we did not identify new codes.

\section{RESULTS}

Of the 4000 surveys mailed out for phase 1 , there were 1072 respondents; of the 603 surveys mailed out for phase 2, there were 464 respondents (Fig. 1). Demographic information of respondents is presented in Table 1.

About $43 \%$ of respondents reported making a major medical treatment decision. Respondents who indicated making a treatment decision were older than respondents who did not (average age 59 years old and 49 years old, respectively). Figure 2 shows the decisions made by respondents. Over half of respondents made decisions about resuscitation, palliative care, or hospice for someone else.

\section{Most Difficult Decision}

Table 2 shows characteristics of the decision indicated most difficult by respondents. The majority were decisions regarding major surgery, made over 3 years ago, and made for a parent. The average age of the patient who needed a decision was 67 years old, but decisions were made for newborns as well as older adults in their nineties.

\section{Decisional Conflict}

The average decisional conflict score was low (24 out of a possible 100), with respondent scores ranging from 0 (no decisional conflict) to 84 (high decisional conflict). Variable selection using cross-validation lasso indicated that three possible predictors showed the lowest mean prediction error and highest $R$-square (Supplemental Table), best fit for assessing the effect on decisional conflict: two aspects of health literacy (ability to actively engage with healthcare providers and understanding health information well enough to know what to do) and the person for whom the decision was made (Table 3 ). Respondents who made the decision for a spouse/partner $(\beta=$ $6.65, p=0.012)$, parent $(\beta=9.27, p<0.001)$, or someone else ( $\beta=10.7, p<0.001)$ had higher decisional conflict. Respondents who reported higher ability to actively engage with healthcare providers $(\beta=-5.24, \mathrm{p}=0.002)$ and to understand health information well enough to know what to do $(\beta=-$ $6.12, p=0.001$ ) had lower decisional conflict.

\section{Qualitative Results}

Of the 464 respondents for phase 2, $390(84 \%)$ responded to at least one open-ended question. We distinguished 3 categories from their responses.

Difficulty in Making End-of-Life Decisions. Many respondents expressed extreme difficulty in making the decision and the enormity of the situation; as one respondent stated, "I had his life in my hands!" Respondents described weighing the risks and benefits of the decision, including the quantity and quality of life. While some respondents expressed difficulty accepting that the end of life was near, others recognized they were "done fighting." A major concern for engaging in treatment was experiencing pain, but there were other repercussions described, for example, "My mother had dementia and her home called to say she was having problems breathing. All I could think about is how traumatic it would be for her to go to the hospital - she died later that day." Some respondents felt confident that they made the right decision whereas others continued to second-guess their choice.

Vivid Memories. Respondents described vivid memories regarding the decision-making process. What one respondent remembered most about a hospice decision for her mom was, "My dad breaking down and saying, 'I don't want to lose her but I also don't want to see her suffer." Another respondent shared, "My nephew sitting there crying and saying that he'll be fine.... And just seeing him not accepting what the doctors were telling us." These vivid memories show the heartbreak and emotion embedded in these decisions that can affect the entire family.

Sources of Support and Frustration. Many respondents stated that knowing the patient's wishes either helped or would have helped families make the decision for a patient. Involving other family members in the decision-making was described by some as supportive and by others as perpetuating conflict. Healthcare practitioners were also a source of support but at times frustration for their care of the patient or for their 


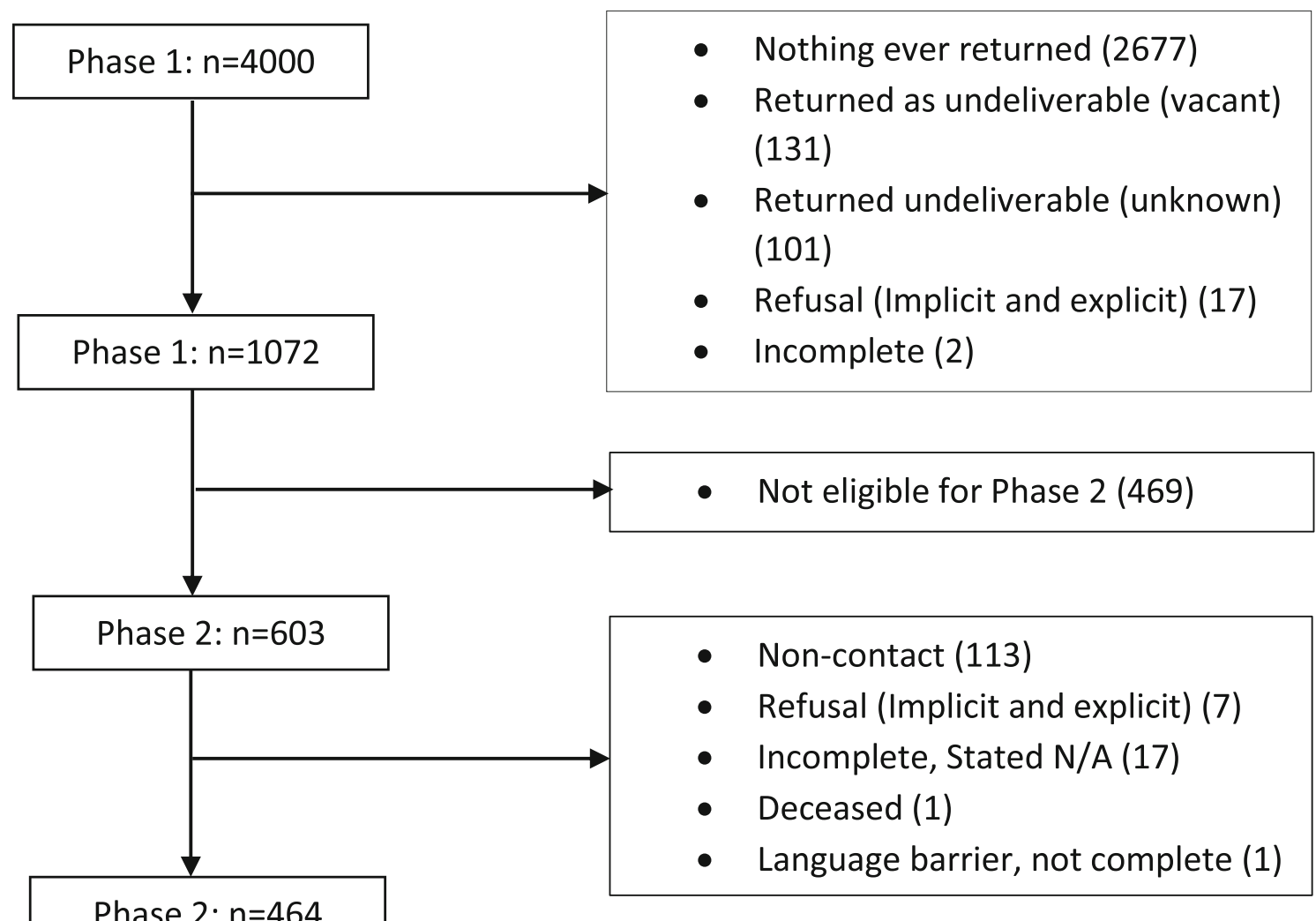

Figure 1 Two-phase enrollment process. Respondents from phase 1 who were eligible were sent the full survey in phase 2.

communication about the patient's situation. One respondent stated, "I think that the American healthcare system conditions us to believe that there is an 'answer' to medical problems and that our doctors have those answers. Most of us know very little about the human body and its workings and therefore place blind trust in the medical profession. When the doctors 'don't know,' we are left with fear, doubt and often suspicion regarding the competency of our providers." Another frustration was the availability of facilities where patients could receive care. One respondent with a loved one in a facility in another state said: "They need the love and support and not be sent to a strange place where they don't know anyone and no

Table 1 Demographic Characteristics of Respondents

\begin{tabular}{|c|c|c|c|c|}
\hline Respondent characteristic & & $\begin{array}{l}\text { Did not make decision, } \\
N(\%)\end{array}$ & $\begin{array}{l}\text { Made decision, } \\
N(\%)\end{array}$ & $\begin{array}{l}\text { Total respondents, } \\
N(\%)\end{array}$ \\
\hline \multicolumn{5}{|l|}{ Gender $(n=1066)$} \\
\hline & Female & $323(30.3)$ & $429(40.24)$ & $752(70.5)$ \\
\hline & Male & $127(11.91)$ & $183(17.17)$ & $310(29.1)$ \\
\hline & Neither female nor male & $3(0.28)$ & $1(0.09)$ & $4(0.04)$ \\
\hline Race/ethnicity $(n=1068)$ & White & $416(38.95)$ & $568(53.18)$ & $984(92.13)$ \\
\hline & African American & $13(1.21)$ & $11(1.03)$ & $24(2.25)$ \\
\hline & Hispanic or Latino & $6(0.56)$ & $10(0.94)$ & $16(1.5)$ \\
\hline & Asian & $7(0.66)$ & $6(0.56)$ & $13(1.22)$ \\
\hline & American Indian or Alaskan Native & $2(0.19)$ & $2(0.19)$ & $4(0.37)$ \\
\hline & Multiple Selected & $6(0.56)$ & $14(1.31)$ & $20(1.87)$ \\
\hline & Other & $3(0.28)$ & $4(0.37)$ & $7(0.66)$ \\
\hline Education $(n=1068)$ & Some high school or less & $16(1.5)$ & $16(1.5)$ & $32(3)$ \\
\hline & Completed high school or GED & $70(6.55)$ & $108(10.11)$ & $178(16.67)$ \\
\hline & Trade school & $14(1.31)$ & $26(2.43)$ & $40(3.75)$ \\
\hline & Some college & $72(6.74)$ & $101(9.46)$ & $173(16.2)$ \\
\hline & Associate's degree or 2-year college degree & $55(5.15)$ & $98(9.18)$ & $153(14.33)$ \\
\hline & Bachelor's degree or 4-year college degree & $149(13.95)$ & $172(16.1)$ & $321(30.06)$ \\
\hline & Master's degree & $60(5.62)$ & $70(6.55)$ & $130(12.17)$ \\
\hline & Advanced degree & $17(1.59)$ & $24(2.25)$ & $41(3.84)$ \\
\hline \multirow[t]{2}{*}{ Age $(n=1060)$} & Mean (SD) & $49.2(18.4)$ & $59.1(15.6)$ & $54.9(17.4)$ \\
\hline & Min, $\max$ & 18,95 & 19,97 & 18,97 \\
\hline
\end{tabular}



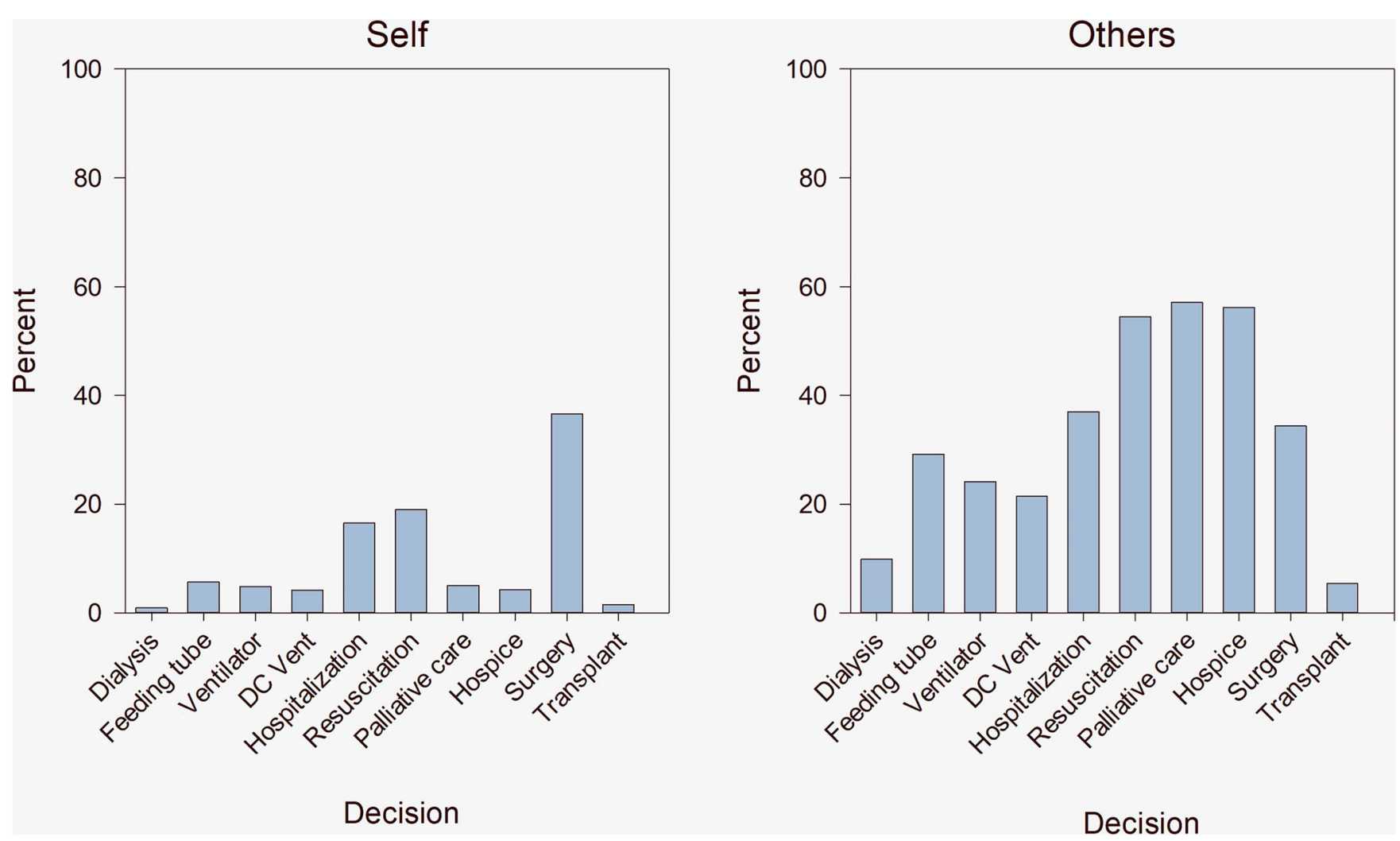

Figure 2 Percent of all respondents from phase 2 who made different types of decisions for themselves ("self") or someone else ("other"). "WD Ventilator" is withdrawing a ventilator, whereas "Ventilator" is putting someone on a ventilator. "Feeding tube" and "Dialysis" include both decisions to begin these treatments and withdraw these treatments.

one to support them at the most difficult time of their life. It's horrible for them. This is the worst thing to have to go through." These descriptions show that frustrations continue even after a difficult decision is made.

\section{DISCUSSION}

About $43 \%$ of respondents made a major medical treatment decision. Most respondents made decisions about resuscitation, palliative care, or hospice for someone else, whereas decisions about major surgery or life support were regarded as the most difficult decisions. Respondents who made the decision for another person (except a child) had higher decisional conflict. Respondents who reported higher ability to actively engage with healthcare providers and to understand health information well enough to know what to do had lower decisional conflict.

A similar study in Canada found that $65 \%$ of residents had made a complex health decision, of which only $0.5 \%$ made a decision about cessation of life support. ${ }^{14}$ Our study had a lower percentage of respondents reporting major medical treatment decisions, but over $20 \%$ had experience discontinuing a ventilator. Differences in findings could be attributed to the types of questions prompted in each study: We asked about the use of specific treatments and care whereas the O'Connor and colleagues' study prompted respondents to consider surgery and medical treatments, along with birth control and lifestyle changes. ${ }^{14}$ The difference in our findings could also be attributed to the time periods of the studies (2019 and 1999). A study of 22 European ICUs found that significantly more limitations in life-prolonging therapies occurred in 2015-2016 when compared with 1999-2000. ${ }^{15}$ As technology continues to advance, it is likely that the need to make major medical treatment decisions will continue to increase. Given that respondents in our study who made treatment decisions were on average 10 years older, the likelihood of needing to make a major medical treatment decision will increase as people age and their parents age (almost half of the most difficult decisions were made for a parent).

Two aspects of health literacy - ability to actively engage with healthcare providers and understanding health information well enough to know what to do-were both statistically significant predictors of decisional conflict. Evidence has shown that individuals with lower health literacy are less likely to ask questions and subsequently engage with healthcare practitioners. ${ }^{16}$ Shared decision-making support tools and strategies can be useful in engaging patients in the conversation, yet Muscat and colleagues found that patients with lower literacy had difficulty answering questions when such tools and strategies were used. ${ }^{17}$ McNeil and Arena have criticized shared decision-making in which the patient still needs to "learn how to speak like a practitioner."18 They propose a model of "harmonics," which seeks to embrace the patient's 
Table 2 Characteristics of the Decision and Decision-maker for the Most Difficult Decision Made

\begin{tabular}{|c|c|c|}
\hline Decision and decision-maker characteristic & & $N(\%)$ \\
\hline \multicolumn{3}{|l|}{ Most difficult decision $(n=401)$} \\
\hline & Major surgery & $101(25.19)$ \\
\hline & Life support & $95(23.69)$ \\
\hline & Hospice & $78(19.45)$ \\
\hline & Cancer treatment & $40(9.98)$ \\
\hline & Code status & $39(9.73)$ \\
\hline & Institutionalization & $25(6.23)$ \\
\hline & Other & $23(5.74)$ \\
\hline \multicolumn{3}{|l|}{ Time since decision $(n=436)$} \\
\hline & $<3$ months & $27(6.19)$ \\
\hline & 3-11 months & $45(10.32)$ \\
\hline & $1-2$ years & $74(16.97)$ \\
\hline & $3-10$ years & $185(42.43)$ \\
\hline & $>10$ years & $105(24.08)$ \\
\hline \multicolumn{3}{|l|}{ Person decision was for $(n=435)$} \\
\hline & Your parent & $200(45.98)$ \\
\hline & Yourself & $90(20.69)$ \\
\hline & Someone else & $67(15.4)$ \\
\hline & Spouse or partner & $59(13.56)$ \\
\hline & Your child & $19(4.37)$ \\
\hline & & Mean (SD), min, max \\
\hline \multicolumn{3}{|l|}{ Health literacy of decision-maker } \\
\hline & Social support for health & $\begin{array}{l}3.29(0.6) \\
1,4\end{array}$ \\
\hline & Appraisal of health information & $3.1(0.55)$ \\
\hline & & 1,4 \\
\hline & Ability to actively engage with healthcare providers & $4.07(0.64)$ \\
\hline & & $1.2,5$ \\
\hline & Understanding health information well enough to know what to do & $4.22(0.58)$ \\
\hline & & $1.6,5$ \\
\hline \multicolumn{2}{|l|}{ Age for whom decision made } & $67.71(21.36)$ \\
\hline & & 0,98 \\
\hline \multirow[t]{2}{*}{ Decisional conflict } & & $24.06(16.77)$ \\
\hline & & $0,84.38$ \\
\hline
\end{tabular}

personal health expertise in the conversation to collaboratively construct meaning. Involving palliative care may also be beneficial; more patients felt heard and understood after palliative care consultation, ${ }^{19}$ suggesting an improvement in communication. Although communication models acknowledge the importance of exploring patient goals and eliciting concerns, ${ }^{20}$ experts have argued for improved understanding of how to tailor such communication to individual patients. ${ }^{21}$ Determining communication strategies that improve engagement of individuals with limited health literacy is warranted.

The vivid memories that some participants described - along with descriptions of difficulty making decisions, secondguessing choices, and concern about loved ones-reveal the psychological burden of these experiences, particularly for families making decisions for patients. The psychological sequelae of family members of patients in the ICU have been documented, ${ }^{22}$ and the cluster of complications from ICU experiences has been recognized as post-intensive care syndrome-family (PICS-F). ${ }^{23}$ However, in our study, many respondents described difficult situations that occurred when the patient was not in the ICU and even when the patient was not in the hospital. Further research should examine the experiences of families who engaged in major medical treatment decision-making in other settings.

This study is limited by the homogenous sample of mostly White respondents with overall high health literacy and low decisional conflict. The respondents were randomly sampled by their address, yet respondents who had higher health literacy and who had made a major medical treatment decision may have been more likely to self-select to participate,

Table 3 Association Between Characteristics of the Decision or Decision-maker and Decisional Conflict

\begin{tabular}{|c|c|c|c|c|}
\hline Decisional conflict & Coefficient & Standard error & $p$ value & $95 \% \mathrm{CI}$ \\
\hline Ability to actively engage with healthcare providers & -5.24 & 1.68 & 0.002 & $-8.54,-1.93$ \\
\hline $\begin{array}{l}\text { Understanding health information well enough to know what to do } \\
\text { Person for whom the decision is made* }\end{array}$ & -6.12 & 1.87 & 0.001 & $-9.79,-2.44$ \\
\hline Your spouse or partner & 6.65 & 2.62 & 0.01 & $1.50,11.80$ \\
\hline Someone else & 10.7 & 2.55 & 0.00 & $5.69,15.71$ \\
\hline Your child & 3.89 & 4.13 & 0.35 & $-4.23,12.01$ \\
\hline Your parent & 9.27 & 1.98 & 0.00 & $5.37,13.16$ \\
\hline
\end{tabular}

*Reference group: Yourself 
creating bias. We also sampled only from one state (Wisconsin), and although it has both urban and rural communities, it may not be generalizable to other populations. In addition, Wisconsin is home to Respecting Choices ${ }^{\circledR}$ and Honoring Choices Wisconsin that have successfully increased advance care planning throughout the state, ${ }^{24-26}$ which may have affected respondents' decisional conflict as well as their overall experience making major treatment decisions.

The need to make a major medical treatment decision for oneself or someone else is likely to increase. We will need to determine tangible communication strategies that encourage patient and family engagement in the conversation, particularly for individuals with limited health literacy. Improving provider-patient and family interactions will be an important mechanism to reduce decisional conflict and the psychological sequelae that result from these experiences.

Acknowledgments: The authors thank the University of Wisconsin Survey Center, University of Wisconsin-Madison.

Corresponding Author: Kristen E. Pecanac, PhD, RN; School of Nursing, University of Wisconsin-Madison, 701 Highland Ave, Madison, WI 53705, USA (e-mail: lund2@wisc.edu).

Funding Information Support for this research was provided by the Office of the Vice Chancellor for Research and Graduate Education at the University of Wisconsin-Madison with funding from the Wisconsin Alumni Research Foundation as well as a 2018 Research Committee Award from the School of Nursing, University of Wisconsin-Madison.

\section{Compliance with Ethical Standards:}

This study was reviewed and approved by the Health Sciences Institutional Review Board. Respondents completing and returning the surveys implied consent

Conflict of Interest: The authors declare that they do not have a conflict of interest.

\section{REFERENCES}

1. American Medical Association. Code of Medical Ethics: Caring for patients at the end of life, 2019, Available at: https://www.ama-assn. org/system/files/2019-06/code-of-medical-ethics-chapter-5.pdf. Accessed Jul 19, 2020.

2. Garvelink MM, Boland L, Klein $\mathbf{K}$, et al. Decisional conflict scale findings among patients and surrogates making health decisions: Part II of an anniversary review. Med Decis Making. 2019;39:315-326.

3. Heyland DK, Heyland R, Dodek P, et al. Discordance between patients stated values and treatment preferences for end-of-life care: Results of a multicentre survey. BMJ Support Palliat Care. 2017;7:292-299.

4. O'Connor AM. User manual-decisional conflict scale, 2010, Available at: https://decisionaid.ohri.ca/docs/develop/User_Manuals/UM_Decisional_Conflict.pdf. Accessed Mar 3, 2020.
5. Silveira MJ, Kim SY, Langa, KM. Advance directives and outcomes of surrogate decision making before death. N Engl J Med. 2010;362:12111218.

6. Chiarchiaro J, Buddadhumaruk P, Arnold RM, White DB. Prior advance care planning is associated with less decisional conflict among surrogates for critically ill patients. Ann Am Thorac Soc. 2015;12;15281533.

7. Orom H, Biddle C, Underwood W 3rd, Nelson CJ, Homish DL. What is a "good" treatment decision? decisional control, knowledge, treatment decision making, and quality of life in men with clinically localized prostate cancer. Med Decis Making. 2016;36:714-25.

8. Kremer H, Ironson G, Schneiderman N, Hautzinger M. "It's my body": Does patient involvement in decision making reduce decisional conflict? Med Decis Making. 2007;27:522-32.

9. Osborne RH, Batterham RW, Elsworth GR, Hawkins M, Buchbinder R. The grounded psychometric development and initial validation of the Health Literacy Questionnaire (HLQ). BMC Public Health. 2013;13;658.

10. Song MK, Sereika SM. An evaluation of the Decisional Conflict Scale for measuring the quality of end-of-life decision making. Patient Educ Couns. 2006;61:397-404.

11. Pecanac KE, Brown RL, Steingrub J, Anderson W, Matthay MA, White DB. A psychometric study of the decisional conflict scale in surrogate decision makers. Patient Educ Couns. 2018;101:1957-1965.

12. Tibshirani R. Regression shrinkage and selection via the lasso. J R Stat Soc Series B Stat Methodol 1996;267-288.

13. Elo S, Kyngas $\mathbf{H}$. The qualitative content analysis process. J Adv Nurs. 2008;62:107-115.

14. O'Connor AM, Drake ER, Wells GA, Tugwell P, Laupacis A, Elmslie T. A survey of the decision-making needs of Canadians faced with complex health decisions. Health Expect. 2003;6:97-109.

15. Sprung CL, Ricou B, Hartog CS, et al. Changes in End-of-Life Practices in European Intensive Care Units From 1999 to 2016. JAMA. 2019:1-12.

16. Aboumatar HJ, Carson KA, Beach MC, Roter DL, Cooper LA. The impact of health literacy on desire for participation in healthcare, medical visit communication, and patient reported outcomes among patients with hypertension. J Gen Intern Med. 2013;28:1469-1476.

17. Muscat DM, Shepherd HL, Morony S, et al. Can adults with low literacy understand shared decision making questions? A qualitative investigation. Patient Educ Couns. 2016;99:1796-1802.

18. McNeil A, Arena R. The evolution of health literacy and communication: Introducing health harmonics. Prog Cardiovasc Dis. 2017:59:463-470.

19. Ingersoll LT, Saeed F, Ladwig S, et al. Feeling heard and understood in the hospital environment: Benchmarking communication quality among patients with advanced cancer before and after palliative care consultation. J Pain Symptom Manage. 2018;56:239-244.

20. Sanders JJ, Curtis JR, Tulsky JA. Achieving goal-concordant care: A conceptual model and approach to measuring serious illness communication and its impact. J Palliat Med. 2018:21:S17-S27

21. Tulsky JA, Beach MC, Butow, PN, et al. A research agenda for communication between health care professionals and patients living with serious illness. JAMA Intern Med. 2017;177:1361-1366.

22. Johnson CC, Suchyta MR, Darowski ES, et al. Psychological sequelae in family caregivers of critically iii intensive care unit patients. A systematic review. Ann Am Thorac Soc. 2019;16:894-909.

23. Davidson JE, Jones C, Bienvenu OJ. Family response to critical illness: Postintensive care syndrome-family. Crit Care Med. 2012;40:618-624.

24. Peltier WL, Gani F, Blissitt J, et al. Initial experience with "Honoring Choices Wisconsin": Implementation of an advance care planning pilot in a tertiary care setting. J Palliat Med. 2017;20:998-1003.

25. Pecanac KE, Repenshek MF, Tennenbaum D, et al. Respecting Choices ${ }^{\circledR}$ and Advance Directives in a Diverse Community. J Palliat Med. 2014;17:282-287.

26. Hammes BJ, Rooney BL. Death and end-of-life planning in one Midwestern community. Arch Intern Med 1998;158:383-390.

Publisher's Note: Springer Nature remains neutral with regard to jurisdictional claims in published maps and institutional affiliations. 